\title{
Balloon pulmonary angioplasty - efficient therapy of chronic thromboembolic pulmonary hypertension in the patient with advanced sarcoidosis - a case report
}

Andrzej Labyk ${ }^{1 *}$ (D), Dominik Wretowski ${ }^{1}$, Sabina Zybińska-Oksiutowicz¹, Aleksandra Furdyna ${ }^{1}$, Katarzyna Ciesielska', Dorota Piotrowska-Kownacka², Olga Dzikowska -Diduch', Barbara Lichodziejewska', Andrzej Biederman²,

Piotr Pruszczyk ${ }^{1}$ and Marek Roik ${ }^{1}$

\begin{abstract}
Background: Approximately a quarter of patients with advanced sarcoidosis develop pulmonary hypertension (PH), which affects their prognosis. We report unusual case of confirmed chronic thromboembolic pulmonary hypertension (CTEPH) in a patient with stage IV sarcoidosis successfully treated with balloon pulmonary angioplasty (BPA).

Case presentation: A 65 years old male with a history of colitis ulcerosa, and pulmonary sarcoidosis diagnosed in 10 years before, on long term oral steroids, with a history of deep vein thrombosis and acute pulmonary embolism chronically anticoagulated was referred to our center due to severe dyspnea. On admission he presented WHO functional class IV, mean pulmonary artery pressure (mPAP) in right heart catheterization (RHC) was elevated to $54 \mathrm{mmHg}$. Diagnosis of CTEPH was definitely confirmed with typical V/Q scan, and with selective pulmonary angiography (PAG) completes by intravascular imagining (intravascular ultrasound, optical coherent tomography). The patient was deemed inoperable by CTEPH team and two sessions of BPA with multimodal approach resulted in significant clinical and haemodynamical improvement to WHO class 11 and mPAP decrease to $27 \mathrm{mmHg}$.
\end{abstract}

Conclusions: Balloon pulmonary angioplasty, rapidly developing method of treatment of inoperable CTEPH patients, is also extremely useful therapeutic tool in complex PH patients.

Keywords: Sarcoidosis, Chronic thromboembolic pulmonary hypertension, Balloon pulmonary angioplasty

\section{Background}

Approximately $26 \%$ of patients with advanced pulmonary sarcoidosis may develop pulmonary hypertension $(\mathrm{PH})$, which is related with poor prognosis $[1,2]$. According to current guidelines, patients with suspected $\mathrm{PH}$ should undergo detailed diagnostic workup and differential diagnosis among others should include chronic lung diseases leading to hypoxia and chronic thromboembolic pulmonary hypertension (CTEPH) [3]. We

\footnotetext{
* Correspondence: endrjulab@tlen.pl

${ }^{1}$ Center for Diagnostics and Treatment of Venous Thromboembolism,

Department of Internal Medicine and Cardiology, Warsaw Medical University,

Infant Jesus Hospital, Lindleya Street 4, 02-005 Warsaw, Poland

Full list of author information is available at the end of the article
}

report an unusual challenging case of CTEPH in a patient with stage IV sarcoidosis.

\section{Case presentation}

A 65 years old male with history of colitis ulcerosa, and advanced sarcoidosis diagnosed 10 years before, on oral steroids was admitted to our department due to exertional dyspnea and right ventricular (RV) heart failure progressing since last 12 months to functional class IV. Two years earlier, he experienced the first severe decompensation of right heart function. At that time $\mathrm{PH}$ was diagnosed on echocardiography. RV to left ventricle (LV) ratio exceeded one (RV:LV - 39/32 mm); peak systolic tricuspid regurgitation gradient (TRPG) was $75 \mathrm{mmHg}$, and decreased tricuspid annular plane systolic excursion

(c) The Author(s). 2018 Open Access This article is distributed under the terms of the Creative Commons Attribution 4.0 International License (http://creativecommons.org/licenses/by/4.0/), which permits unrestricted use, distribution, and 
(TAPSE) $15 \mathrm{~mm}$ indicated significant pressure overload and RV systolic dysfunction. Chest computed tomography $(\mathrm{CT})$ revealed sarcoidosis progression, however no pulmonary thromboemboli were detected. After typical heart failure treatment with diuretics, ACE inhibitors, and steroid dose increase the patient improved and he was discharged home in a good clinical condition in WHO functional class II, with the diagnosis of PH due to sarcoidosis stage IV. One year later, due to acute dyspnea, worsening of RV function and unilateral leg swelling he underwent another chest CT, which this time showed fresh thrombi in the left segmental upper lobe pulmonary artery. Moreover, acute deep vein thrombosis was detected with lower limb compression ultrasound. Long term oral anticoagulation with rivaroxaban was started. Two years later the patient was referred to our center due to progressive functional deterioration. On admission he was in WHO functional class IV, saturation on room air was $85 \%$, blood pressure 120/ $80 \mathrm{mmHg}$, heart rate 90 beats per minute. Mild peripheral edema was present. Distance covered in 6 min walk test (6MWT) was $100 \mathrm{~m}$ with desaturation to $77 \%$. Plasma natriuretic peptide (NT-pro-BNP) concentration was elevated to $6239 \mathrm{pg} / \mathrm{ml}$ (normal range $<125 \mathrm{pg} / \mathrm{ml}$ ). Echocardiography showed severe RV pressure overload with TRPG $95 \mathrm{mmHg}$, dilatation of both right atrium and ventricle. Preserved morphology and function of LV was observed. Chest CT scan showed signs of advanced interstitial lung fibrosis (Fig. 1a) and calcified mediastinal lymph nodes (Fig. 1b). However, organized thrombi in both pulmonary were also detected (Fig. 1c and d). At that time multifactorial etiology of $\mathrm{PH}$ was considered: sarcoidosis with secondary $\mathrm{PH}$ and local in situ thrombosis, or CTEPH in a patient with stage IV sarcoidosis, and with deep vein thrombosis in the past. Lung perfusion scan with SPECT/CT showed bilateral perfusion defects which strongly suggested thromboembolic component of $\mathrm{PH}$. Right heart catheterization (RHC) followed by selective pulmonary angiography (PAG) showed mean pulmonary artery pressure (mPAP) of $54 \mathrm{mmHg}$. Pulmonary artery wedge pressure (PAWP) was $6 \mathrm{mmHg}$ and pulmonary vascular resistance (PVR) was 13,5 Wood Units. Selective PAG confirmed chronic thromboembolic lesions suggestive of CTEPH - left upper lobe artery occlusion, intravascular webs in right pulmonary artery (Fig. 2a). After experienced cardiac surgeon consultation, the patient was deemed inoperable due to advanced, sarcoidosis - related lung changes and propable complications regarding the use of extracorporeal circulation. He was finaly qualified for balloon pulmonary angioplasty (BPA).

BPA procedures were performed according the previously published protocol [4]. Importantly, in order to confirm intraluminal localization of thromboembolic

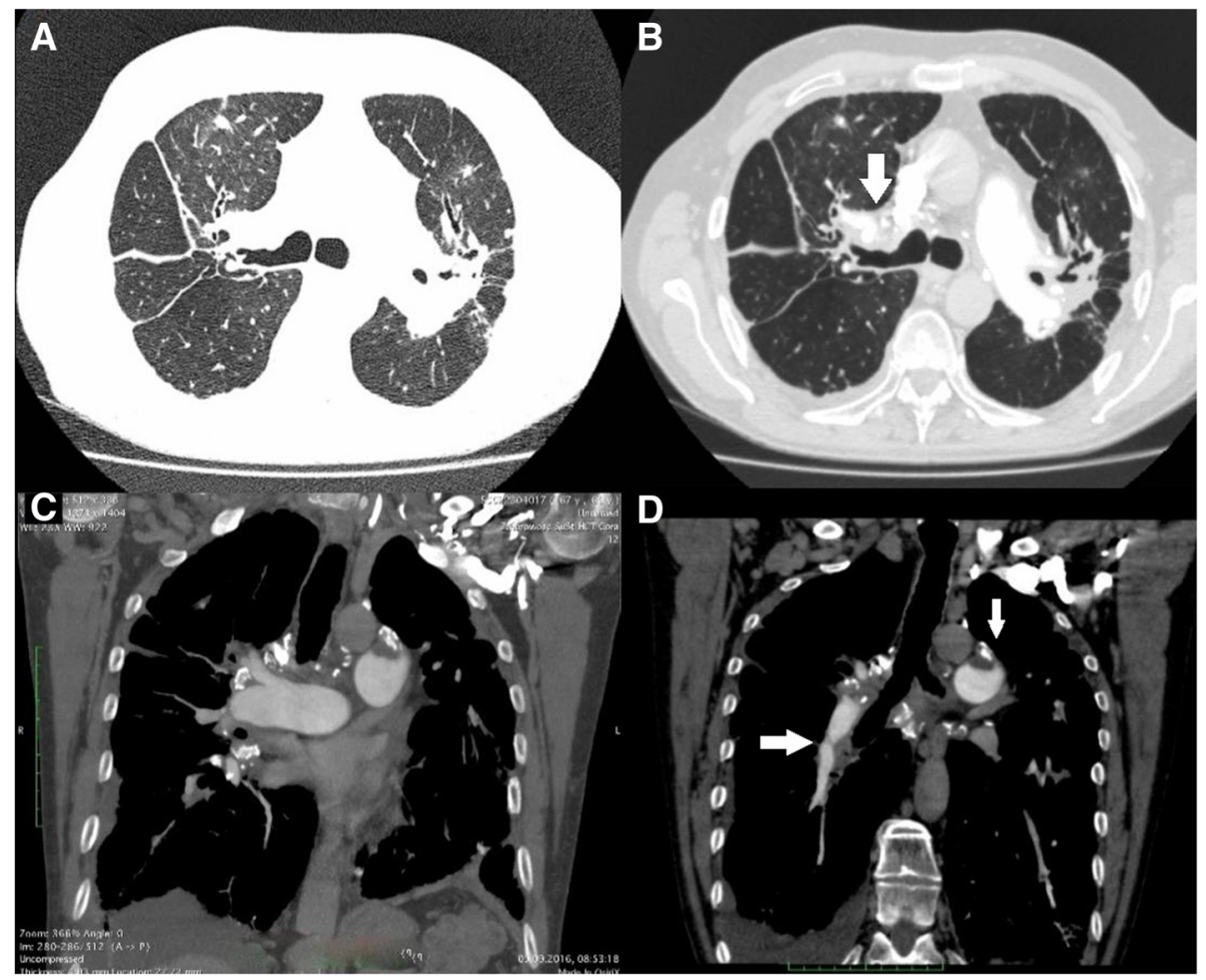

Fig. $\mathbf{1}$ a, b - interstitial lung fibrosis and calcified lymph nodes. $\mathbf{c}, \mathbf{d}$ - organized thrombi in left and right pulmonary arteries 


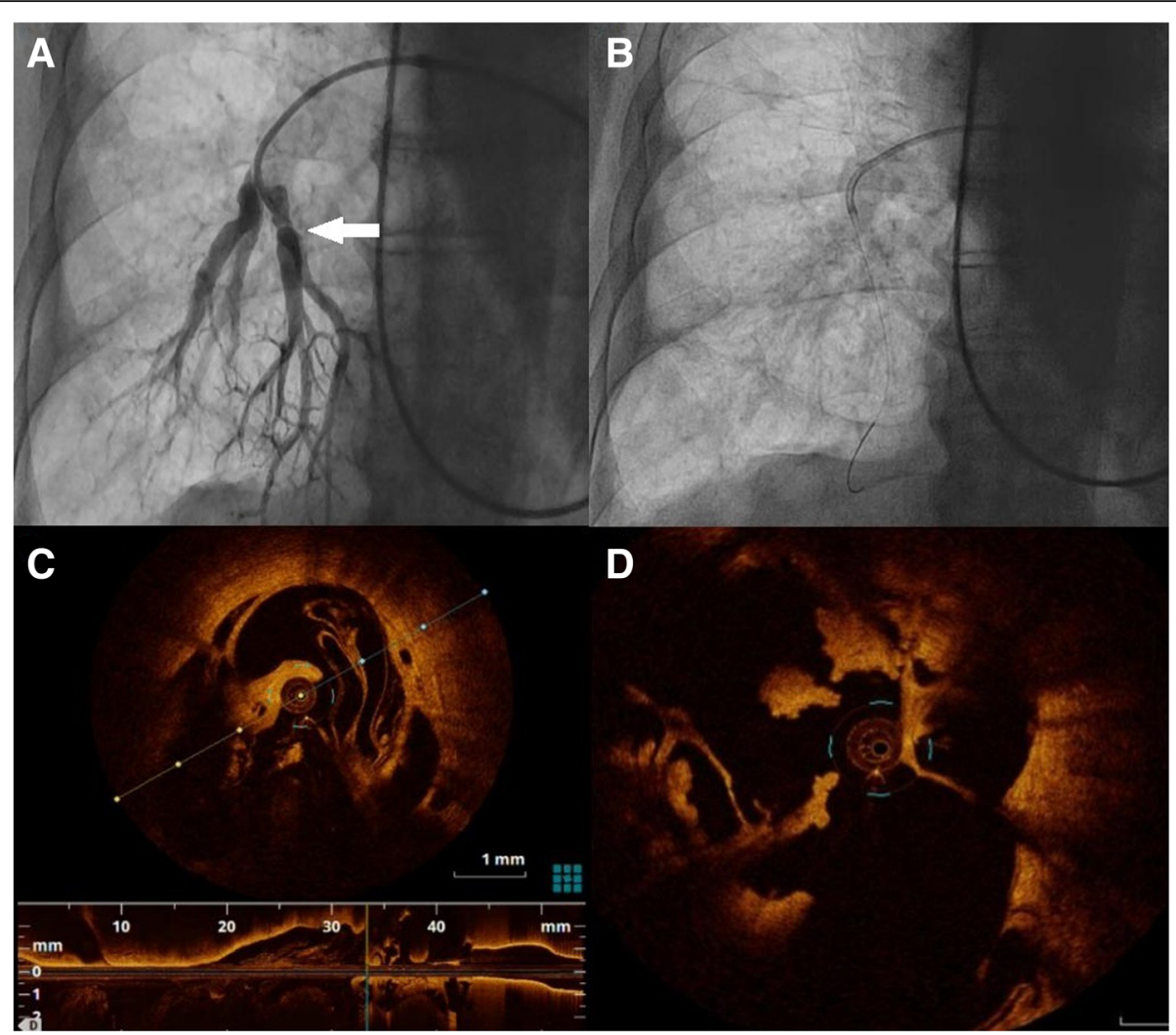

Fig. 2 a - initial selective right pulmonary artery angiography with intravascular web marked with arrow. b, c - intravascular web/organized thrombus in optical coherent tomography $(\mathrm{OCT})$. $\mathbf{d}$ - guide wire with pressure catheter to assess pulmonary pressure ratio (PPR)

lesions intravascular imaging with optical coherence tomography (OCT) and intravascular ultrasound (IVUS) were performed. It allowed to definitely confirm organized thrombi (Fig. 2a - white arrow, 2C, 2D). With the use of pressure catheter we assessed hemodynamic significance of intrapulmonary lesions (Fig. 2b). Pulmonary pressure ratio (PPR, the ratio of the pressure distal to the lesion (Pd) divided by the pressure proximal to the lesion (Pp)) was assessed to optimize the BPA procedure and to minimize potential complications such as reperfusion pulmonary injury (RPI). PPR in arteries A9 and A10 was 0.19 and 0.22 - which suggested functional occlusion of both arteries. After simultaneous inflation of balloon catheters - "kissing balloon" technique $(4.0 \times 20 \mathrm{~mm}$ and $4.0 \times 27 \mathrm{~mm}$ respectively), followed by proximal optimization with $7.0 \times 30 \mathrm{~mm}$ balloon catheter resulted in PPR 0.63 in A9 and 0.65 in A10 (Fig. 3, part A and D - green curve marking distal pressure). There were no periprocedural complications. Mean PAP after the first BPA procedure decreased from $52 \mathrm{mmHG}$ to $40 \mathrm{mmHg}$. The second BPA session was performed 3 weeks later (left pulmonary artery), also without complications. Both procedures (3 segmental lesions in total) resulted in further hemodynamic and functional improvement, with mPAP drop to $27 \mathrm{mmHg}$ at 12 months follow - up. Patient was still in functional class II WHO, echocardiography and 6MWT showed further improvement in clinical condition and RV function (Table 1).

\section{Discussion and conclusions}

Sarcoidosis related $\mathrm{PH}$ as is predominantly caused severe lung fibrosis or by compression of pulmonary arteries by mediastinal fibrosis and lymphadenopathy $[1,2,5]$. Pulmonary artery stenting was reported to be efficient in the treatment of pulmonary arteries compression in advanced sarcoidosis [5], while pharmacology with sildenafil or other $\mathrm{PH}$ specific dugs are of very limited short term efficacy $[6,7]$.

Presented case is probably one of the first available reports with initially suspected sarcoidosis related $\mathrm{PH}$, which eventually was verified by pulmonary angiography and multimodal intravascular assessment as CTEPH. There are no data on optimal treatment in such challenging patients. After being deemed inoperable due to advanced sarcoidosis resulting in severe lung alterations, the patient was qualified to BPA procedure. Percutaneous catheter based method, is a rapidly developing therapeutic method for CTEPH patients. There is a growing evidence that in expert centers it is safe and effective, allowing normalization or near normalization of 

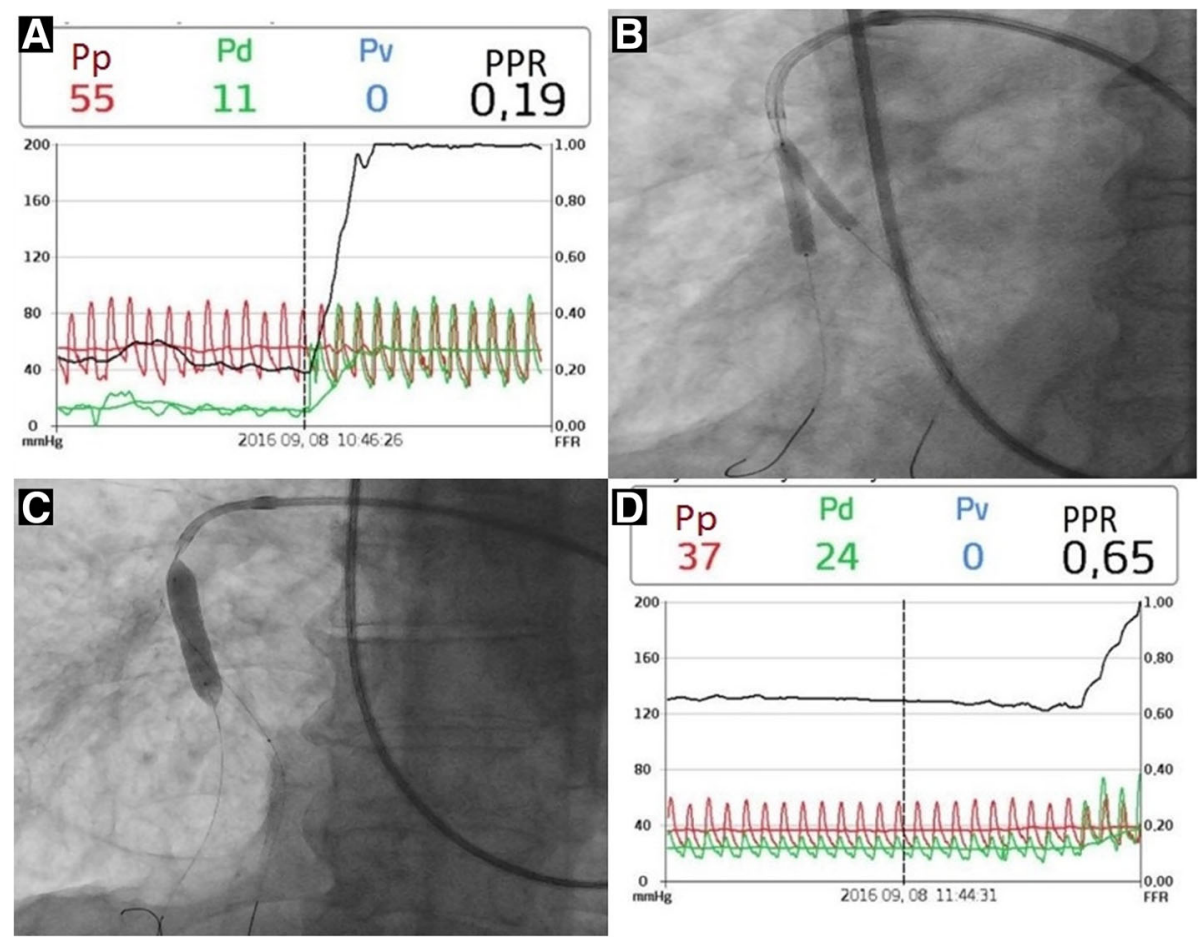

Fig. 3 a - PPR assessment before balloon pulmonary angioplasty, green curve nearly flat at first with rise after thrombus crossing. $\mathbf{b}$ - "kissing balloons" technique. c - proximal optimalization with large balloon catheter. $\mathbf{d}$ - final PPR assessment, green curve showes restored pressure on safe level, preventing reperfusion pulmonary injury

mPAP in majority of treated cases $[4,8-13]$. In our institution we have started BPA program in inoperable CTEPH in 2014. BPA procedures are performed according to the previously described protocol [4] with the use of pressure catheter, and in selected cases with OCT/ IVUS intravascular imaging. Our data indicate that this

Table 1 Clinical and hemodynamical assessment before and after 2 BPA sessions

\begin{tabular}{lll}
\hline & Before treatment & At 12 months follow-up \\
\hline WHO functional class & IV & $\|$ \\
NT-pro-BNP level (pg/ml) & 6239 & 281 \\
6MWT distance (m) & 100 & 405 \\
TRPG (mmHg) & 95 & 31 \\
RVSP (mmHg) & 102 & 53 \\
TAPSE (mm) & 14 & 20 \\
ACT (ms) & 63 & 100 \\
mPAP (mmHg) & 54 & 27 \\
Cl (I/min/m ${ }^{2}$ ) & 2.08 & 3.34 \\
RAP (mmHg) & $14 / 10 / 9$ & $5 / 3 / 1$ \\
systolic/diastolic/mean & & 3.7 \\
PVR (Wood Units) & 13.5 &
\end{tabular}

NT-pro-BNP natriuretic peptide concentration, $6 M W T 6$ min walk test, TRPG tricuspid regurgitation peak gradient, RVSP right ventricle systolic pressure, TAPSE tricuspid annular plane excursion, AcT acceleration time, mPAP mean pulmonary artery pressure, $\mathrm{Cl}$ cardiac index, $R A P$ right atrial pressure, $P V R$ pulmonary vascular resistance, $B P A$ balloon pulmonary angioplasty technique is safe and effective also in CTEPH high risk patients with severe comorbidities [14]. We are convinced that multimodal approach not only allowed to confirm CTEPH definitely in the patient with advanced sarcoidosis, but it also allowed to treat him and successfully and safely. We performed angioplasties of selected thromboembolic lesions, which were proved to be hemodynamically significant in multimodal assessment. Such fast improvement was also observed in other CTEPH patients treated with BPA. Based on our experience, it is related with initial treatment of the lesions in segmental artreries of lower lobes. In presented case, specific drug therapy (riociguat) was not avaliable at that time. Patient was on sildenafi ( $25 \mathrm{mg}$ t.i.d.) and initial mPAP decreased only by $2 \mathrm{mmHg}(54 \mathrm{mmHg}$ in initial RHC versus $52 \mathrm{mmHg}$ before first BPA session). Thus, only two BPA sessions resulted in mPAP reduction and significant clinical improvement. We think that BPA became a real therapeutic option in CTEPH patients, who are not candidates for surgery.

\footnotetext{
Abbreviations

6MWT: 6 min walk test; AcT: Acceleration time; BPA: Balloon pulmonary angioplasty; Cl: Cardiac index; CT: Computed tomography; CTEPH: Chronic thromboembolic pulmonary hypertension; IVUS: Intravascular ultrasound; LV: Left ventricle; mPAP: Mean pulmonary artery pressure; NT-proBNP: Natriuretic peptide concentration; OCT: Optical coherent tomography; PAG: Pulmonary angiography; PAWP: Pulmonary artery wedge pressure; PH: Pulmonary hypertension; PPR: Pulmonary pressure ratio; PVR: Pulmonary
} 
vascular resistance; RAP: Right atral pressure; RHC: Right heart catheterization; RPI: Reperfusion pulmonary injury; RV: Right ventricle; RVSP: Right ventricle systolic pressure; SPECT: Single-photon emission computed tomography; TAPSE: Tricuspid annular plane excursion; TRPG: Tricuspid regurgitation peak gradient

\section{Funding}

The authors declare that there was no funding regarding this manuscript.

\section{Availability of data and materials}

Data sharing is not applicable to this article as no datasets were generated or analysed during the current study.

\section{Authors' contributions}

$\mathrm{AL}$ - analysis and interpretation of patient's data, second operator in BPA procedures, leading contribution in writing the manuscript. DW, MR - first operators in BPA procedures, data analysis and interpretation, supervision, contribution in writing the manuscript. SZO, AF, KC - diagnostic work-up, follow-up, data collection. DPK - radiology studies assessment. ODD, BL echocardiography assessment. $\mathrm{AB}$ - cardiac surgeon, consulted the patient. PP - contribution in writing the manuscript, supervision. All authors read and approved the final manuscript.

\section{Ethics approval and consent to participate}

Not applicable.

\section{Consent for publication}

Written informed consent for publication in BMC Pulmonary Medicine was obtained.

\section{Competing interests}

The authors declare that they have no competing interests.

\section{Publisher's Note}

Springer Nature remains neutral with regard to jurisdictional claims in published maps and institutional affiliations.

\section{Author details}

'Center for Diagnostics and Treatment of Venous Thromboembolism, Department of Internal Medicine and Cardiology, Warsaw Medical University, Infant Jesus Hospital, Lindleya Street 4, 02-005 Warsaw, Poland. ²Department of Radiology, Infant Jesus Hospital, Lindleya Street 4, 02-005 Warsaw, Poland. ${ }^{3}$ Cardiac Surgery Department Medicover Hospital, Rzeczypospolitej 5 Avenue, 02-972 Warsaw, Poland.

Received: 9 March 2018 Accepted: 20 July 2018

Published online: 16 August 2018

References

1. Boucly A, Cottin V, Nunes $H$, et al. Management and long-term outcomes of sarcoidosis-associated pulmonary hypertension. Eur Respir J. 2017. https:// doi.org/10.1183/13993003.00465-2017.

2. Baughman RP, Shlobin OA. Treatment of sarcoidosis-associated pulmonary hypertension: so close, and yet so far. Eur Respir J. 2017; https://doi.org/10 1183/13993003.01725-2017

3. Galiè N, Humbert M, Vachiery JL, et al. 2015 ESC/ERS Guidelines for the diagnosis and treatment of pulmonary hypertension: The Joint Task Force for the Diagnosis and Treatment of Pulmonary Hypertension of the European Society of Cardiology (ESC) and the European Respiratory Society (ERS): Endorsed by: Association for European Paediatric and Congenital Cardiology (AEPC), International Society for Heart and Lung Transplantation (ISHLT). Eur Heart J. 2016;37(1):67-119.

4. Roik M, Wretowski $D$, Łabyk $A$, et al. Refined balloon pulmonary angioplasty driven by combined assessment of intra-arterial anatomy and physiologyMultimodal approach to treated lesions in patients with non-operable distal chronic thromboembolic pulmonary hypertension--Technique, safety and efficacy of 50 consecutive angioplasties. Int J Cardiol. 2016:203:228-35.

5. Hamilton-Craig CR, Slaughter R, McNeil K, et al. Improvement afte angioplasty and stenting of pulmonary arteries due to sarcoid mediastinal fibrosis. Heart Lung Circ. 2009;18(3):222-5.
6. Toonkel RL, Borczuk AC, Pearson GD, et al. Sarcoidosis-associated fibrosing mediastinitis with resultant pulmonary hypertension: a case report and review of the literature. Respiration. 2010;79(4):341-5.

7. Kacprzak A, Szturmowicz M, Burakowska B, et al. Sarcoidosis-associated pulmonary hypertension treated with sildenafil - a case report. Adv Respir Med. 2017;85(5):258-63.

8. Mizoguchi H, Ogawa A, Munemasa M, et al. Refined balloon pulmonary angioplasty for inoperable patients with chronic thromboembolic pulmonary hypertension. Circ Cardiovasc Interv. 2012;5(6):748-55.

9. Kataoka M, Inami T, Hayashida K, et al. Percutaneous transluminal pulmonary angioplasty for the treatment of chronic thromboembolic pulmonary hypertension. Circ Cardiovasc Interv. 2012;5:756-62.

10. Sugimura K, Fukumoto $Y$, Satoh K, et al. Percutaneous transluminal pulmonary angioplasty markedly improves pulmonary hemodynamics and long-term prognosis in patients with chronic thromboembolic pulmonary hypertension. Circ J. 2012;76(2):485-8.

11. Inami T, Kataoka M, Shimura N, et al. Pressure-wire-guided percutaneous transluminal pulmonary angioplasty: a breakthrough in catheterinterventional therapy for chronic thromboembolic pulmonary hypertension. JACC Cardiovasc Interv. 2014;7(11):1297-306.

12. Roik M, Wretowski $D$, Łabyk A, et al. Optical coherence tomography of inoperable chronic thromboembolic pulmonary hypertension treated with refined balloon pulmonary angioplasty. Pol Arch Med Wewn. 2014;124(12): 742-3.

13. Roik M, Wretowski D, Rowiński O, et al. Refined balloon pulmonary angioplasty in inoperable chronic thromboembolic pulmonary hypertension--a multi-modality approach to the treated lesion. Int J Cardiol. 2014;177(3):e139-41.

14. Roik M, Wretowski $D$, Łabyk A, et al. Refined balloon pulmonary angioplastya therapeutic option in very elderly patients with chronic thromboembolic pulmonary hypertension. J Interv Cardiol. 2017;30(3):249-55.

\section{Ready to submit your research? Choose BMC and benefit from:}

- fast, convenient online submission

- thorough peer review by experienced researchers in your field

- rapid publication on acceptance

- support for research data, including large and complex data types

- gold Open Access which fosters wider collaboration and increased citations

- maximum visibility for your research: over $100 \mathrm{M}$ website views per year

At BMC, research is always in progress.

Learn more biomedcentral.com/submissions 